\title{
La comunicación del Tercer Sector y el compromiso de los jóvenes en la era digital
}

\author{
Hirugarren sektorearen komunikazioa \\ eta gazteen konpromisoa aro digitalean
}

\author{
Third sector's communication \\ and youth commitment in the digital era
}

\section{Carmen García Galera ${ }^{1}$ \\ Cristóbal Fernández Muñoz ${ }^{2}$ Jesús del Olmo Barbero ${ }^{3}$}

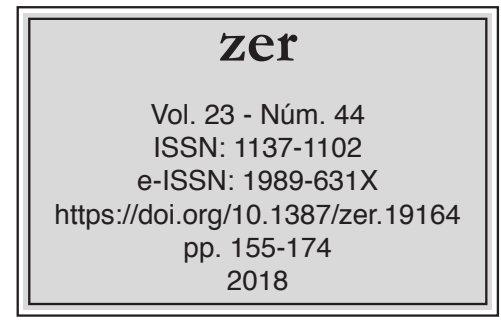

Recibido el 9 de abril de 2018, aceptado el 30 de abril de 2018.

\section{Resumen}

Las organizaciones del Tercer Sector han encontrado en las redes sociales una herramienta de comunicación para llegar e implicar de forma más eficaz a los jóvenes. En este artículo, se aborda el papel que la comunicación digital está desempeñando en las relaciones de las organizaciones no gubernamentales (ONG) con estos públicos. Mediante la técnica del grupo de discusión con responsables de comunicación de distintas ONG, se constata la necesidad de mejorar y adaptar sus estrategias de comunicación recurriendo a nuevos enfoques y técnicas propias del internet social, como el empleo de celebridades, youtubers o bloggers.

Palabras clave: jóvenes; ONG; comunicación; digital; participación.

\section{Laburpena}

Hirugarren sektoreko erakundeek sare sozialak erabiltzen dituzte gazteengana iristeko eta gazteak modu eraginkorragoan inplikatzeko komunikazio tresna gisa. Artikulu honetan aztertzen da komunikazio digitalak zer-nolako garrantzia duen gobernuz kanpoko erakundeek (GKE) publiko horrekin dituzten harremanetan. Hainbat GKEetako komunikazio arduradunekin eztabaida taldeen teknika erabiliz, egiaztatu da beren komunikazio estrategiak hobetu

\footnotetext{
Universidad Rey Juan Carlos, carmen.garcia@urjc.es

2 Universidad Complutense de Madrid, cristfer@ucm.es

3 Universidad Rey Juan Carlos, jesus.delolmo@urjc.es
} 
eta egokitu beharra dutela eta Internet sozialari dagozkion ikuspegi eta teknikak erabili, hala nola pertsona ospetsuak, youtuberrak eta blogerrak.

Gako-hitzak: gazteak; GKE; komunikazioa; digitala; parte-hartzea.

\begin{abstract}
The Third Sector organizations have found in social networks a communication tool to reach and involve young people in the most effective way. This article addresses the important role that digital communication is playing in the relations of non-governmental organizations (NGOs) with these audiences. Through the technique of the discussion group with communication directors from different NGOs, it is found that it is necessary to improve and adapt their communication strategies targeted to young people including new approaches and techniques part of the social internet, such as the use of celebrities, youtubers or bloggers.
\end{abstract}

Keywords: young people; NGOs; communication; digital; participation. 


\section{Introducción}

\subsection{La comunicación digital en las organizaciones}

Internet en general, con sus medios online, sus redes y medios sociales, está significando un cambio incuestionable en el mundo de la comunicación empresarial (Berceruelo, 2016). Que una organización viva ajena a los medios sociales en pleno siglo XXI sería el equivalente a haberle dado la espalada a la radio y la televisión en la segunda mitad del XX (Mariñas, 2016). Las redes sociales, toda la web 2.0 en su conjunto, constituyen un ecosistema nuevo aún. Un ecosistema es una comunidad de seres vivos cuyos procesos vitales se encuentran interrelacionados. El desarrollo de estos seres vivos se produce en función de los factores físicos de este ambiente compartido. Si trasladamos este concepto al entorno digital, se puede afirmar que un ecosistema digital reproduce los mecanismos de los ecosistemas naturales a un entorno virtual, en el que sus integrantes se relacionan y comparten online (Zafra, 2012). Es una metáfora utilizada para ejemplificar la manera en la que funcionan y se integran los diferentes elementos y herramientas que forman parte de la estrategia digital de una organización (Santa, 2015).

Como señala López (2005), en este ecosistema hay tres elementos protagonistas: las tecnologías digitales, los nuevos medios o medios sociales surgidos a raíz de estas tecnologías, y el público, elemento indispensable para el establecimiento de las interrelaciones. Por lo tanto, parece que toda organización vive en la actualidad en un ecosistema relacional singular, distinto al de décadas pasadas, en donde existe mayor dinamismo, interrelación e influencia mutua entre los distintos agentes - frente al carácter estático, parcelado y de mayor independencia de los distintos actores en el pasado- (González, 2015).

Es precisamente el público, como consecuencia de la digitalización, el elemento en el que se ha producido un mayor cambio en su percepción por las organizaciones y en su forma de relacionarse. Tradicionalmente, los programas de comunicación corporativa tan sólo consideraban a los colectivos a los que se dirigían sus acciones de comunicación como agentes finales, meros receptores de un mensaje determinado, sin que existiera - o la hubiera de forma muy limitada - retroalimentación alguna y, mucho menos, una relación personal estructurada con los individuos que lideraban o componían dichos colectivos (González, 2015).

En numerosas ocasiones la dificultad para obtener esa retroalimentación estribaba en que se utilizaban medios de comunicación masiva - como la prensa impresa - para hacerles llegar los mensajes. En otras - como en comunicaciones directas a inversores o analistas mediante newsletters o herramientas similares - se adaptaba y segmentaba en mayor medida el mensaje, pero no existía un diálogo cara a cara, persona a persona, basada en los intereses mutuos de la organización y los individuos de ese colectivo.

Las redes sociales han supuesto un cambio sin precedentes. Imponen rapidez en la respuesta y, además, que la actitud de la organización sea conversacional (Mariñas, 2016). Los nuevos canales de comunicación aportan inmediatez y permiten llegar a muchos públicos a los que antes era mucho más lento o difícil llegar. Pero ahora esos públicos son más participativos y la organización tiene que ser capaz de conversar con ellos (Berceruelo, 2016). 
Antes de la llegada de las redes sociales, según plantea Bernal (2015), la agenda de los medios de comunicación era construida únicamente en base a lo que los periodistas consideraban noticiable. Por lo tanto, el lector solo podía acceder a esa agenda única - el medio no ofrece sino esa versión, una versión de la realidad según su línea editorial - , pasiva, cerrada - no permite participación en la elección de temas - y limitada - ofrece una única visión, es una fuente como medio - . Las redes sociales han permitido una transición a una agenda múltiple — tantas opciones como usuarios que tengan un perfil en la red y elijan a quien seguir - , activa - el usuario elige y participa - , abierta - pueden participar incluso personas que no sean seguidas - y colaborativa - como consecuencia de la participación y de la opción de crear y compartir contenidos - .

\subsection{Las ONG en el medio digital}

Este ecosistema digital definido hasta aquí necesita de una adaptación progresiva de las organizaciones, incluyendo en este contexto las organizaciones no gubernamentales (ONG). Las ONG tienen presencia en los medios sociales con una triple finalidad: hacerse ver entre la población, generar conciencia sobre los asuntos que defienden y, en tercer lugar, convertir esos medios en un canal para encauzar las aportaciones de los ciudadanos, que resultan fundamentales para la subsistencia de sus objetivos. Según el estudio realizado por la Universidad de Massachusetts en 2014, casi todas las organizaciones benéficas y sin fines de lucro de Estados Unidos estaban ya presentes en las redes sociales. El $98 \%$ de ellas utilizaba al menos una plataforma social. De todas las redes sociales, YouTube era la herramienta social más usada con un 97\% de los casos, seguida de Facebook con un $92 \%$, Twitter con un $86 \%$ y Pinterest con un $72 \%$ (marketingdirecto.com, 2014). En ese mismo estudio, el $81 \%$ de los administradores de las redes sociales de las ONG aseguraron que hacerse conocidas era el objetivo principal por el que las usaban, seguido a cierta distancia del $40 \%$ de organizaciones que afirmaban que estaban en redes sociales para obtener donaciones.

Así las cosas, en el contexto 2.0, no es que hayan aparecido nuevas situaciones, nuevos contextos o nuevos objetivos de comunicación para las organizaciones del Tercer Sector. Lo que ocurre es que las redes sociales y las plataformas se han convertido en una herramienta útil y necesaria que les van a ayudar a difundir su misión, aumentar la cantidad de donantes, reclutar voluntarios, ser referentes en una temática, generar alianzas, concienciar sobre un tema, incidir en políticas públicas o comunicarse con los destinatarios, entre otros.

La comunicación digital parece haber cambiado la manera tradicional de funcionar y de comunicar de las ONG como organizaciones que persiguen un fin de ayuda social y lo hacen sin ánimo de lucro, de forma independiente de gobiernos y administraciones. El campo de actuación de las organizaciones no gubernamentales es difícil de clasificar, dada la diversidad de causas que defienden (Brinkerhoff y Brinkerhoff, 2002). Meriläinen y Vos (2011) definen el papel de estas organizaciones como un poder compensatorio del poder tradicional, que se crea mediante la movilización del apoyo público y la presentación de cuestiones sensibles a la opinión pública y a los políticos. Para estos mismos autores, cuántas más personas se sientan atraídas o apoyen la causa iniciada por la organización, más fuerza tendrá ante aquellos que toman las decisiones. 
Es el público, por tanto, quien puede desarrollar o colaborar a un proceso de cambio (Goss, 2001), por lo que las ONG han animado desde siempre a la participación de los ciudadanos: tradicionalmente, con cartas, firmas de peticiones en mesas petitorias, boicots, u otras tácticas comunicativas (Scholte, 1999). Por lo tanto, si hasta hace poco eran necesarios los medios de comunicación tradicionales como instrumento único para llegar a grandes audiencias y conseguir el cambio social que proclamaban, las redes sociales y el universo digital les ha proporcionado una nueva herramienta para llegar a sus públicos sin intermediarios.

Como proclaman Meriläinen y Vos (2011), las redes han propiciado un cambio en las estrategias de comunicación, que invita a los ciudadanos a participar directamente, y que da paso al denominado activismo social.

El Informe global sobre tecnología online de las $O N G$ (2017) elaborado por la publicación Nonprofit Tech for Good, recoge información del uso que las organizaciones del tercer sector realizan de las redes sociales como herramienta para llegar a su público objetivo. Según exponen en su página web, es el único proyecto de investigación anual que se dedica a estudiar la manera en que las ONG utilizan la tecnología online a escala global. En esta última edición, han recogido información de 4.907 ONG de 153 países de todo el mundo. Algunos datos de interés muestran que el $92 \%$ de las ONG tienen un perfil en Facebook, el $72 \%$ un perfil en Twitter y el 39\% un perfil en Instagram (Nonprofit tech for good, 2017).

Por lo tanto, si bien el fondo y la funcionalidad de las ONG no han cambiado, la digitalización les ha puesto sobre la mesa una fórmula de comunicación globalizada, que llegaría de manera sencilla, rápida, sin fronteras, a todos los ciudadanos del planeta, a todas las personas que, finalmente, son capaces de producir cambios, de hacer posible lo imposible, haciendo lo que los gobiernos no pueden o no tienen intención de hacer (Simmons, 1998).

El trabajo realizado por Meriläinen y Vos (2011), mencionado con anterioridad, sobre cómo la comunicación digital ha cambiado las formas de llegar a la ciudadanía de las organizaciones no gubernamentales, concluye, entre otras cuestiones, que las plataformas se actualizan constantemente, que las redes sociales proporcionan información de manera casi inmediata sobre sus actividades invitando a los usuarios a acceder a sus sitios web para obtener una información más detallada. La comunicación digital permite a las ONG crear conciencia en la ciudadanía sobre las cuestiones que abordan según sus temáticas y colocarlas en la agenda social

El estudio realizado por Lovejoy y Saxton (2012) sobre la comunicación en redes sociales de las organizaciones no gubernamentales presenta tres objetivos de esta comunicación: informar, formar comunidad y actuar. En su estudio, realizado entre las 100 ONG más grandes de Estados Unidos, concluyen que enviar información al público objetivo es importante para estas organizaciones, y plataformas como Twitter ayudan a hacerlo, de manera rápida y eficaz, con solo 280 caracteres. Los usuarios, sin embargo, pueden decidir cuánto quieren saber leyendo solo el tuit o cliqueando en el enlace para saber más. La segunda función, crear comunidad, implica diálogo, y aquí es donde empieza el verdadero compromiso, cuando las redes online se desarrollan y los usuarios pueden unirse a la conversación y proporcionar retroalimentación a las organizaciones. 
La tercera categoría es la acción. En esta categoría es donde los usuarios no sólo sienten que están marcando una diferencia, sino que empiezan a hacer algo al respecto, ya sea presentándose en un evento, firmando una petición o haciendo una donación. Quizás sea el principal objetivo de las organizaciones y las redes sociales están proporcionando una nueva vía para esa acción.

Conceptos como el de clickactivismo o slacktivism ("activismo de sofá") han surgido de la mano de las redes sociales, y hacen sentir en sus usuarios que son ciudadanos activos ante situaciones sociales que demandan su participación. El trabajo de García, Fernández y Porto (2017) profundiza en ambos conceptos y en las ventajas e inconvenientes de esta forma de movilización. No obstante, los usuarios quieren información y formar parte del diálogo, pero una organización de este tipo cumple su misión haciendo que sus seguidores hagan algo por la causa que apoyan.

\subsection{Los jóvenes se movilizan}

Los jóvenes ya no acuden a los medios tradicionales de comunicación para conocer qué está ocurriendo en el mundo. De hecho, su principal medio para estar al tanto de lo que ocurre son las redes sociales. En este sentido, como declara Joaquín Moral a raíz de los atentados de 2015 en París, la irrupción de las redes sociales ha cambiado radicalmente la forma de vivir estas desgracias. El cambio puede apreciarse tanto a nivel informativo, con un alcance inmediato y contado a tiempo real, con capacidad para solidarizarse y rectificar errores, como logrando que las personas se impliquen más (Cabanelas, 2015). De hecho, ante acontecimientos de índole social o política que importan e impactan a la población en general, los jóvenes utilizan las redes sociales para informarse, participar y movilizar (García, Fernández y Porto, 2017), hasta tal punto que los medios tradicionales se hacen eco de esas conversaciones dada la repercusión que tiene la movilización online.

González-Anleo (2005: 59) afirmaba hace más de quince años que "en la mayoría de las investigaciones sociológicas sobre la juventud española llama la atención su apatía política, su débil participación en grupos y movimientos de carácter social y la ausencia de una ética o cultura cívica, elementos esenciales de lo que llamamos ciudadanía”.

Frente a este escenario cargado de pesimismo, el estudio "Jóvenes, Satisfacción Personal, Participación Asociativa y Voluntariado" realizado por el Observatorio del Instituto de la Juventud (2014) evidencia una situación en la que se incrementa el número de jóvenes que se implican en acciones asociativas y cívico-participativas. Así, el estudio muestra que el $38 \%$ de los jóvenes encuestados pertenecían a alguna asociación en el momento en el que se realizó la encuesta, lo que supone un incremento del $9 \%$ si se compara con los datos registrados por este mismo observatorio en 2007.

Es cierto que los espacios asociativos orientados a las actividades deportivas, lúdicas y/o recreativas tienen más peso entre la gente joven que las asociaciones de carácter político o social (Injuve, 2014), pero no debemos olvidar que el cauce de actuación de la sociedad civil se identifica con el tejido asociativo. Las asociaciones se conciben como la primera alternativa "horizontal" a la participación política.

Es interesante señalar que la variable sexo ofrece algunas diferencias en la participación en asociaciones. Así, las asociaciones deportivas, recreativas y estudiantiles 
cuentan con una mayor participación de hombres, mientras que las asociaciones religiosas, culturales y benéficas tienen una mayor presencia de mujeres.

Las actividades de voluntariado que resultan más interesantes para los jóvenes españoles son aquellas relativas a la infancia y la juventud (17\%), de ayuda a la pobreza (12\%), relativas a la salud y la sanidad (11\%), de trabajo con discapacitados $(10 \%)$ y la ecología y el medioambiente $(8 \%)$. Le siguen las actividades de ayuda al Tercer Mundo y países en conflicto (7\%), con drogodependientes y alcohólicos (7\%), con ancianos (5\%), de apoyo a la mujer (4\%) y las actividades culturales y de recuperación del patrimonio (4\%). Entre 2006 y 2014, período en el que la economía entró en crisis, se produjeron cambios en la escala de actividades de voluntariado más atractivas para los jóvenes, aumentando el interés mostrado en actividades relacionadas con la ayuda a la pobreza, la salud y la sanidad, y disminuyendo el interés en actividades del ámbito medioambiental y de ayuda al tercer mundo.

En las asociaciones, los jóvenes buscan emplear el tiempo en actividades que les gustan (40\%), mientras que las razones finalistas tienen menos peso en los motivos para participar. En menor medida, entre los motivos que incitan a la gente joven a participar en una asociación se encuentran aspectos como "sentirse útil ayudando a los demás" (12\%), "disfrutar de los motivos de ser asociado" (11\%), el hecho de sentirse incluidos en el grupo de iguales "porqué los amigos pertenecen a la asociación" $(10 \%)$ o "para estar con personas que piensan como tú" $(9 \%)$, y en último lugar para "poder defender los derechos y las opiniones $(8,5 \%)$.

$\mathrm{El}$ interés por el asociacionismo queda patente en el dato de que uno de cada cuatro jóvenes que no ha pertenecido nunca a una asociación declara que le gustaría participar en alguna. Los jóvenes españoles señalan que una de las principales ventajas de estar asociado sería hablar y compartir ideas y hacer nuevas amistades, lo que pone de relieve el carácter socializador del asociacionismo juvenil.

En cuanto a actividades de voluntariado, igualmente los jóvenes tienen una alta participación. Cuatro de cada diez jóvenes españoles declara tener experiencia en actividades de voluntariado. El $9 \%$ de la población encuestada manifestaba colaborar en ese momento con alguna entidad de voluntariado, perfil que corresponde en mayor medida con mujeres, jóvenes con niveles de estudios superiores, estudiantes y solteros. El informe de Injuve constató igualmente que en los últimos años se ha incrementado el número de jóvenes que colabora habitualmente con organizaciones de voluntariado.

Los jóvenes en España tienen entre sus principales motivaciones para hacerse voluntario la realización de un servicio social a la comunidad $(90 \%)$, la sensación de sentirse útil (88\%), las creencias morales (82\%), la interacción con la gente (79\%) y las creencias religiosas $(60 \%)$.

Estos patrones de actitudes y comportamiento de los jóvenes españoles no distan de los de otros países. En cierta medida, al igual que ha ocurrido con la nueva sociedad digital en la última década, el fenómeno de la globalización no es ajena al ámbito de la movilización social y política de los jóvenes. Así, las acusaciones de que los jóvenes son políticamente apáticos y que de alguna manera han fracasado en su deber de participar en muchas sociedades democráticas en todo el mundo han sido refutadas por un número creciente de académicos en los últimos años (Loader, 2007; Marsh, O’Toole y Jones, 2007; Loader, Vromen y Xenos, 2014). 
En los últimos tiempos emergen también propuestas de nuevas vías de participación que se caracterizan por una concreción mayor de la forma y objetivo de la participación. Así, Kallio y Häkli (2013) señalan que los jóvenes, al igual que otros colectivos, pueden estar facultados para participar en asuntos que les conciernen y así obtener un terreno más firme como miembros de sus comunidades y sociedades, si se les da la oportunidad.

Igualmente, cada vez son más los estudios que, como el de Yamamoto, Kushin y Dalisay (2013), señalan que la expresión política online modifica el panorama tradicional de la participación cívica, mejorando los efectos de los medios tradicionales, dado el rol movilizador de las redes sociales en el proceso del desarrollo de la ciudadanía digital activa de los jóvenes.

\section{Metodología}

El objetivo general de esta investigación es conocer cómo las organizaciones no gubernamentales utilizan la comunicación digital como medio necesario para movilizar y llamar a la participación a los jóvenes. Como objetivos específicos, el estudio trata de reconocer las acciones de éxito de las ONG para la movilización de los jóvenes, así como las ventajas y dificultades que estas organizaciones encuentran en el uso de las redes sociales como herramienta para llegar a este público objetivo.

Entre las preguntas de investigación que tratamos de responder figuran: (1) ¿qué hacen las Organizaciones del Tercer Sector para tratar de movilizar a los jóvenes y hacerlos partícipes de sus causas?; (2) ¿qué ventajas encuentran estas organizaciones en las redes sociales?; y (3) ¿qué tácticas han tenido éxito para llegar e implicar a los jóvenes y por qué?

Ante este planteamiento metodológico, la hipótesis de investigación es que las organizaciones del Tercer Sector, conscientes de la importancia que la comunicación digital tiene en la vida de los jóvenes, tratan de utilizar los medios digitales para conseguir el compromiso de este colectivo con las acciones solidarias que desarrollan. No obstante, estas organizaciones, acostumbradas a acciones de comunicación tradicionales, experimentan cierta resistencia y desconocimiento de las fórmulas y herramientas digitales que consigan atraer al mayor número de jóvenes a sus eventos.

En este contexto, el grupo de discusión se presenta como la técnica más adecuada de obtención de información sobre el objeto de estudio. Se trata de una técnica cuya meta es la recolección del máximo de información posible, en un tiempo preestablecido, sobre las percepciones o actitudes del conglomerado de personas que se ha pretendido representar mediante la formación de ese grupo en particular (de Miguel, 2005). Producen un tipo de datos que difícilmente podrían obtenerse por otros medios, ya que sitúan a los participantes en situaciones reales y naturales en las que es posible la espontaneidad y en la que, gracias al clima permisivo, surgen opiniones, sentimientos, deseos personales que en situaciones experimentales rígidamente estructuradas no serían manifestadas.

El colectivo seleccionado objeto de estudio es el que hemos definido como "representantes de Organizaciones del Tercer Sector u Organizaciones No Gubernamentales"4. Respondiendo a la capacidad operativa de recolección y análisis,

${ }^{4}$ GRUPO DE DISCUSIÓN. Ficha técnica. Estudio cualitativo realizado mediante grupos de discusión. 
como sugieren Hernández Sampieri, Fernández Collado, \& Baptista Lucio (2006) se seleccionaron a individuos responsables de acciones de comunicación dentro de alguna de las siguientes ONG o plataforma web: Greenpeace, Unicef, Fundación Garrigou, Cooperación Internacional, Asociación Española contra el Cáncer (AECC) y Change.org. Las variables de segmentación utilizadas en la selección de las ONG fueron, en primer lugar, organizaciones con páginas web y perfiles en redes sociales en la que se pueda participar en campañas, donativos, denuncias, etc.; en segundo lugar, organizaciones que pertenecieran al ámbito online, offline o a los dos; y, por último, organizaciones cuyo ámbito de actuación pudiera ser local, nacional y/o internacional. El objetivo es la riqueza, profundidad y calidad de la información, no la cantidad ni la estandarización. Se trata de una muestra orientada hacia la investigación cualitativa diversa o de máxima variación, que se utiliza cuando se busca mostrar distintas perspectivas y representar la complejidad del fenómeno estudiado, o bien, documentar diversidad para localizar diferencias y coincidencias, patrones y particularidades (Hernández, Fernández y Baptista, 2006).

La sesión fue grabada y transcrita para proceder posteriormente al análisis de contenido de la información, asistido con la utilización del programa de análisis de datos cualitativos Atlas.ti (v.7.5.6.). La información obtenida fue analizada con la técnica del micro-análisis para el posterior establecimiento de conceptos. Así, los textos se analizaron por párrafos independientes, fueron fragmentados en unidades de significado y clasificados en códigos cerrados consensuados previamente, y en códigos abiertos creados al hilo del proceso. La información fue finalmente ordenada en los códigos y familias de códigos. Se trata de un procedimiento habitual para el análisis del discurso obtenido mediante esta técnica cualitativa de investigación (García y Fernández, 2016)

\section{Análisis de resultados}

\subsection{Las posibilidades de las redes sociales}

Existe un elevado consenso entre los representantes de las organizaciones del Tercer Sector en que las críticas al individualismo o despolitización actual de la juventud, como principal obstáculo para su participación ciudadana, no son acertadas. Estas organizaciones parten del supuesto de que son los jóvenes los que quieren participar en causas sociales pero que son las organizaciones las que tienen dificultades para llegar a ellos e implicarlos:

"No sabemos darles propuestas de valor que les resulten atractivas, o no sabemos llegar a ellos. Yo lo que sí veo es que es más problema de las organizaciones. No creo que ellos no se quieran implicar. De hecho, los ves participando en un montón de cosas... Yo creo que incluso más que en

Esta investigación, ha sido desarrollada dentro del programa Provuldig sobre Vulnerabilidad Digital, Ref: S2015/HUM-3434 llevado a cabo por un consorcio de cinco grupos de investigación consolidados en las Universidades madrileñas CEU San Pablo, Rey Juan Carlos y Complutense de Madrid (CES Villanueva). Lugar de realización: Madrid. Fecha de realización del trabajo de campo: Primavera, 2016. 
generaciones anteriores. El consumo colaborativo está a la orden del día. No tengo tan claro que ellos estén despolitizados o individualizados".

"Sí, bueno, les cuesta, pero sí quieren oír, sí quieren oír cómo se hacen las cosas, sí quieren ser buenas personas, sí quieren tener valores. Sí les revienta que los políticos roben, sí están en eso. Cómo ellos lo manifiestan o cómo ellos lo expresan es diferente, claro, a lo que nosotros hacemos. Igual no creen que el voto sirva para nada, entonces hay que trabajar en eso. Pero no es que pasan de todo y no les interese nada".

Ante esta situación, las ONG ven todo un mundo de posibilidades en la comunicación social digital, en concreto, en las redes sociales, como herramienta para llegar a los jóvenes y movilizarlos. Representan, a este respecto, un novedoso y eficaz canal de comunicación transversal y de participación de sus públicos objetivos, que, además, a las organizaciones "ofrecen una oportunidad de oro para fomentar la participación y sobre todo la comunicación".

Así, entre las ventajas y valoraciones que surgen en sus discursos se encuentran, en primer lugar, el papel de información que las redes sociales tienen, en general, para sus usuarios, en tanto en cuanto, las redes permiten a las organizaciones mantener informadas de sus acciones a sus comunidades y potenciales seguidores. Información de todo aquello que hacen las organizaciones, de sus campañas y de sus llamamientos a la participación:

"Y luego ellos, pues hay muchas veces que si no no se enteran, o sea, la comunidad no se entera de muchas acciones que se llevan a cabo por parte de la organización si no es a través de un Facebook que les va alimentando cada día de temas interesantes. Las organizaciones hacemos mucho, muchísimas cosas y la gente tiene que saber lo que hacemos y las redes sociales son una herramienta increíble para eso".

En este sentido, las redes sociales promueven una comunicación más eficaz, en la medida en que abren un hueco que permite a las organizaciones colarse en las redes sociales de sus audiencias, entre los contenidos de las vidas cotidianas de sus potenciales seguidores, en su día a día más personal y privado:

"Yo creo que también es una forma de entrar, de que ellos reciban tus mensajes incluso de una manera más eficaz que puede ser una web o un medio de comunicación tradicional en el sentido de que te cuelas un poco entre los contenidos de su vida diaria, de su interés, de sus amistades. La Red es una fuente de consulta prácticamente diaria por otros motivos, y te dan una ventana, una apertura para aparecer entre esos contenidos que son como de información más personalizada, la que realmente les interesa, te abren un hueco". 
Esta cualidad lleva de la mano otra, que las organizaciones mencionan, y que hace referencia a la velocidad a la que puede propagarse una información o la propia llamada a la participación de una organización sea muy elevada:

"El hecho de que cualquier mensaje se pueda propagar rápidamente es algo que es muy positivo tanto si es la red, nuestro caso, como si eres la organización que utilizas la red para llevar a cabo tu acción".

Otra de las cualidades que las organizaciones no gubernamentales otorgan a la comunicación digital, en concreto, a las redes sociales es el carácter directo y la bidireccionalidad de la comunicación. Es decir, las redes sociales permiten también a las organizaciones testar la información que difunden y valorar su impacto a través del feedback directo que pueden recibir de los usuarios en la forma de opiniones, evaluaciones, críticas o sugerencias acerca de sus diversas iniciativas y campañas de participación:

\begin{abstract}
"Puedes llegar a la gente, lanzar tus mensajes, tus campañas, y tener un feedback inmediato. Te sirve también para evaluar el interés de tus seguidores, o de la gente con la que tienes relación. Es un termómetro para ver qué es lo que realmente les gusta, les interesa, en la medida en que tú puedes recoger ese feedback, esa opinión. Eso es de enorme interés para las entidades porque te sirve para evaluar lo que realmente les está llegando".
\end{abstract}

Para las ONG, las redes sociales representan también un importante canal para sensibilizar y comprometer a los jóvenes en la resolución de problemas sociales. Las campañas de sensibilización e información y participación que lanzan las organizaciones a través de las redes sociales online persiguen el objetivo de concienciar a los jóvenes desde temprana edad, de hacerles reflexionar, de abrirles la mente sobre cuestiones de interés social, de empoderarlos para comprometerlos en cambiar el mundo y afrontar los graves problemas que detectan y que afectan a todos:

"Invertimos mucho esfuerzo en la formación desde la infancia, porque a la larga, lo que no se siembra, luego es difícil de cosechar”.

"Es un caldo de cultivo que después se va hacer más mayor. No nos importa nada que estén en esta u otra ONG. Por el medio ambiente, por el cáncer, por la gente que tiene causas individuales y que cambien el mundo. Es decir, abrir esa cabeza".

"Esta generación $Z$, es una generación en la que los valores son importantes. O sea, más que otra cosa. Quiero decir, sus valores de ahora, que serán los valores que tendrán dentro de cinco o diez años. 
Tanto ONG como plataformas de peticiones web, persiguen con su actuación empoderar a los jóvenes, transmitirles confianza y motivación, tratando de implicarlos y de hacerles protagonistas del cambio social:

"Nuestro trabajo tiene que ser más empoderar, para que ellos lo sepan contar a su manera, y en sus canales. Igual más que comunicar como hasta ahora".

"Transmitirles también la confianza en que ellos pueden ser protagonistas de ese cambio y transmitirles esa motivación en que realmente pueden hacer mucho".

Las ONG también parten de una cierta autocrítica en tanto que consideran que existe entre ellas un desconocimiento del nuevo contexto comunicativo de los jóvenes y de las redes sociales online donde se encuentran e interactúan los jóvenes, posiblemente diferentes de aquellas que utilizan los adultos. Actualmente, se percibe un esfuerzo de los responsables de las ONG para comprender los nuevos usos y conocer los medios a través de los cuales los jóvenes establecen procesos comunicativos online: por entender lo que está ocurriendo y cómo funciona:

"Ponernos un poco a su nivel, intentando también entender lo que está ocurriendo. Entonces, nosotros estamos básicamente intentando dirigirnos a sus canales y no forzar la adopción de tu mensaje. Sencillamente decir: "Bueno, conócelo". No consumen televisión, no consumen radio, consumen YouTube, que es un mundo en el que te viene el youtuber y te pide tres mil euros para hacerte una mención en un vídeo. Entonces, es un poco entender cómo funciona eso... O sea, por qué tú no puedes convertirte en un youtuber".

"Por lo que podemos ver, sí que son seres sociales, lo que pasa es que igual no están en las mismas redes que nosotros".

Los testimonios también hablan de la dificultad de las organizaciones a la hora de ofrecer a los usuarios más jóvenes propuestas de valor que les resulten atractivas

"Lo único que hace falta es que... pues se les tienda un poco la mano. Pero, cuando se les ofrece algo bueno, desde luego que lo captan y lo valoran, yo creo".

"No sabemos llegar a ellos".

"A ellos no les interesan las cosas como se las decimos nosotros".

"Cómo ellos lo manifiestan o cómo ellos lo expresan es diferente, claro, a lo que nosotros hacemos".

"Pero yo lo que siento es que a ellos no les interesan las cosas como se las decimos nosotros, no que no les interesen". 
Por último, las organizaciones del Tercer Sector conciben las redes sociales como un "nuevo cauce de entrada" en la saturada agenda de los medios tradicionales. Ofrecen la posibilidad a las organizaciones de insertar sus temas candentes, de forma indirecta, en la agenda de los medios ya que estos se suelen hacer eco de aquellas cuestiones [trending topics] que acaban siendo virales o destacados en las redes sociales online

"es otra vía de propagación muy importante, ¿no? pues de manera tradicional la puerta de entrada a los medios de comunicación llega un momento en que no es tan fácil, ¿no? ya están sobresaturados y de esta manera pues, es otra vía de llegada".

\subsection{Acciones de comunicación de las ONG en redes sociales}

A pesar de las dificultades, las ONG aluden a las iniciativas que promueven desde las redes sociales online para animar a la participación, adoptando diferentes estrategias y tácticas para incentivar la participación de los más jóvenes. Entre las iniciativas citadas por los representantes de las organizaciones se encuentran, en primer lugar, el llamamiento a la participación a través de acciones o propuestas lúdicas. Se trata de adaptar las campañas al público al que se dirigen: sensibilizar, fomentar la solidaridad e informar a través de una propuesta de participación que adopte la forma de acción que los jóvenes identifiquen relacionada con lo lúdico - gamificación - y con la obtención de alguna contrapartida, resultado, premio o regalo

“puede ser un juego, una propuesta de 'gamificación', mandar un contenido, en otra una acción..."

Otra de las acciones está relacionada con el lanzamiento de encuestas a través de las redes sociales que sirven como gancho, es decir, van asociadas a las peticiones de solidaridad o colaboración que realizan las plataformas web y las ONG. Estas encuestas sirven para despertar la curiosidad de los usuarios como medio para informar sobre un problema y para tratar de obtener colaboración ciudadana:

"Por ejemplo, el uso de encuestas de Twitter te puede servir para crear conciencia sobre una determinada cuestión de una manera distinta. Mostrabas tres opciones y decías, 'si quieres saber la respuesta, la tienes en la petición'. Entonces mucha gente, por el hecho de 'voy a intentar adivinar', te respondía a la petición. Ese tuit tuvo un engagement de 15 o 20 veces mayor que un tuit normal, conseguías que fueran a la petición para ver la respuesta y ya de paso, si te interesa el tema, pues puedes firmar". 
Para incentivar a los jóvenes a la participación, las ONG coinciden en que parece necesario incluir la dimensión emocional en la elaboración de las historias que sirven de base a las campañas de participación. En opinión de las ONG, el recurso actual al storytelling emocional - construcción de un relato emocional-que toque la fibra sensible de los potenciales participantes en sus campañas, es una acción que ha venido funcionando bien a la hora de animar a la participación, especialmente juvenil:

"Tienes que contar una historia que toque un poco la fibra sensible. Porque siempre ha sido así, pero, vamos, cada vez yo creo que va más por ahí, contar historias".

Se trata de una modalidad de marketing social que trata de huir de los mensajes institucionales clásicos de las organizaciones que llamaban directamente a la colaboración, por una nueva narrativa que, a través de lo emocional, permita la identificación del público con el problema que se presenta:

"El 'necesitamos tu ayuda', en último lugar. Antes hay mucho camino que recorrer para llegar a tocar un poco el corazoncito con las historias con contenido emocional con las que la gente se puede identificar".

Uno de los problemas que plantea esta estrategia para las ONG es que, cada vez más, este recurso está siendo utilizado en exceso, tanto desde el ámbito de la publicidad como desde las plataformas web de peticiones:

"Cada vez hay más competencia en este sentido; siempre ha sido clásico en las ONG. Ha sido nuestro mensaje, pero cada vez lo utilizan más sectores y más medios de comunicación”.

Las ONG están de acuerdo cuando hacen referencia a la necesidad de recurrir también a valores universales como leit motiv en sus campañas, con el propósito de llamar al compromiso, al voluntariado y a la participación. Se trata de la transmisión de mensajes en las campañas online que reflejen valores universales con los que todo el mundo se puede identificar, tales como la honestidad, la transparencia, la lucha contra la corrupción o la preocupación por los demás. Estos mensajes, en opinión de las organizaciones, tienen un importante eco entre los potenciales participantes. Su impacto ayuda a alcanzar los objetivos de sensibilización y de difusión de información previstos, ya que se trata de valores con los que las personas quieren identificarse y quieren que los demás le vean a él: 
"Reflejan valores con los que todo el mundo se puede identificar, y que cualquiera está encantado de hacerlos suyos, de transmitírselos a sus contactos, porque se identifica con ellos".

"Todo el mundo está encantado de darte un retweet o de que la gente lo identifique con esos valores tan positivos".

En opinión de las organizaciones, resulta especialmente interesante ofrecer como ejemplo en estas campañas el testimonio de jóvenes, de gente normal, de personas que encarnan estos valores y con quienes los jóvenes se puedan sentir identificados:

"Hay que mostrar los valores encarnados en otros jóvenes como ellos y que les puedan servir de referencia".

"Es gente normal y corriente, con muchísimo atractivo, gente real. Que no es un anuncio, que al protagonista lo puedes conocer pasado mañana, que te cuenta lo que está haciendo y lo contento que está; yo creo que el valor del ejemplo tira mucho"

A pesar de la importancia que ven en las redes sociales para conseguir la movilización juvenil para sus causas, en opinión de las ONG y plataformas web, el planteamiento de campañas que combinan actuaciones offline con acciones online resulta más efectivo que aquel que utiliza solo uno de los dos canales para su difusión:

"A nosotros, las campañas que mejor nos han funcionado son las que han sido armadas tanto online como offline".

A este respecto se considera que actualmente ambos canales son complementarios a la hora de diseñar las campañas.

"El trabajo entre los dos ámbitos es muy complementario, porque la capacidad de saltar de uno al otro, dependiendo de la campaña, dependiendo del tema, dependiendo de miles de variables es muy elevada, o sea, por lo menos nosotros creemos que la capacidad de saltar de uno a otro es muy grande".

\subsection{El papel de los influenciadores}

En este contexto digital y participativo, los denominados influenciadores juegan un papel determinante para llamar a la acción offline o para la vinculación online: el 
recurso a líderes, a creadores de opinión - youtubers, bloggers - para informar de forma alternativa acerca de sus campañas y de los problemas que plantean, al público joven. Algunas ONG recurren a los youtubers y bloggers, pero también a artistas famosos - celebrities - , como fórmula para difundir sus campañas y también para hacer llegar sus mensajes a audiencias juveniles.

Se trata de líderes de opinión en quienes confían los jóvenes, se convierten en referentes por el prestigio adquirido en su trayectoria de actividad online, avalada por los miles de seguidores registrados en sus cuentas o canales de YouTube. Con estos "fichajes" las ONG aprovechan la capacidad de estas personas de llegar a amplias audiencias juveniles, cuyo acceso sería imposible de alcanzar a través de los mensajes institucionales de las organizaciones:

"Estamos trabajando varias fórmulas, y una que nos funciona ahora es trabajar con influenciadores, lo que buscamos es youtubers".

"Trabajamos con gente que detectamos que tiene una sensibilidad especial, no solamente sirve que lo contemos nosotros, sino que te lo cuente alguien en quien confías, una blogger que te gusta, una instagramer que sigues".

"Tienen otra forma de comunicarse. Y esta gente hace así, y tiene cinco mil 'me gustas' en una foto levantado con el pelo girado. Sí, sí, sí, ¡cinco mil "'me gustas"! Es que para conseguir cinco mil 'me gustas' tienes que hacer la obra del Escorial. Nuestra ONG, como organización, no puede sacar un tweet y tener esa repercusión en redes. A ese nivel y con esas edades, ¡no!’.

A la hora de elegir a los protagonistas de sus campañas, se buscan personas a quienes, según los responsables de las $\mathrm{ONG}$, se les atribuyen las siguientes características: en primer lugar, que estén dotados de una sensibilidad, un estilo especial, una forma diferente de llegar a los jóvenes; y en segundo lugar, que se muestren interesados por las campañas que promueven las ONG y estén dispuestos a ofrecer de forma desinteresada su colaboración:

“Cero euros, ¿eh? No hemos pagado un duro. Lo hacen de manera voluntaria”.

Si bien, no ocurre así en todos los casos. El reclutamiento por parte de las organizaciones de líderes de opinión para sus campañas, no siempre es posible de forma desinteresada ya que algunos bloggers y youtubers exigen una compensación económica a cambio de su implicación:

"A nosotros los blogueros si nos cobran, o sea, nos quieren cobrar y no les pagamos, por eso no tenemos blogueros, pero las youtubers, por ejemplo, si colaboran y no nos cobran". 
"Intentamos ponernos a su nivel intentando también entender lo que está ocurriendo: no consumen televisión, no consumen radio, consumen YouTube. Es un mundo en el que te viene el youtuber y te pide tres mil euros para hacerte una mención en un vídeo y es como ¡noooo!”.

En general, se considera que la comunicación que establecen con los potenciales participantes juveniles en sus campañas, es más efectiva si no lo cuentan solamente las organizaciones, sino también estas personas que son de su generación, quienes tienen una forma especial y diferente de escenificar sus mensajes, una narrativa que permite comunicarse y conectar con los jóvenes en su mismo lenguaje, con sus valores, preocupaciones y problemas, estilos de vida, formas de hacer y de decir:

"Ellas [ - las influenciadoras - ] no lo van a contar como nosotros lo contamos; su lenguaje no es el nuestro. Tienen esa forma de contar las cosas, de llegar a la gente. Y sólo a través de ellos se puede hacer".

"La manera en que lo comunica [el influenciador] no tiene nada que ver pues con otra visión. Entonces, lo que están haciendo es [establecer una comunicación especial]: 'oye, es mi estilo de vida, ¡cómo molo!, adopta mi estilo de vida".

\section{Conclusiones}

Las organizaciones del Tercer Sector han entrado de lleno en el ámbito digital. Estas organizaciones han encontrado en las redes sociales un espacio para la participación cívica, para informar sobre sus objetivos solidarios, incrementar su notoriedad o, incluso, aumentar la cantidad de donantes que mantienen viva a la organización. Así pues, como se refleja en ste trabajo, las redes sociales y la comunicación digital en general, permite a estas organizaciones mantener informadas de sus acciones a sus comunidades y potenciales seguidores, destacando en especial, la velocidad a la que puede propagarse rápidamente esta información así como el carácter directo y la bidireccionalidad de la comunicación que es posible establecer en ellas entre organizaciones y potenciales participantes. Adicionalmente, la comunicación digital se convierte en un medio para amplificar los mensajes de las organizaciones, en la medida en que cada usuario de una red social online, se convierte, en un potencial altavoz o difusor alternativo de la información que recibe de las organizaciones a través de su red de contactos.

Se podría, pues, añadir que la comunicación digital permite en este contexto una democratización relativa de los canales o vías de comunicación existentes hasta el momento, sin olvidar que es una herramienta que se convierte en un importante canal para sensibilizar y comprometer a los jóvenes en la resolución de problemas sociales. No obstante, las organizaciones del Tercer Sector manifiestan también que, a pesar de encontrar un aliado en las redes sociales para la sensibilización ante la 
injusticia o el empoderamiento ciudadano, también siguen encontrando problemas y obstáculos para incentivar o promover la participación juvenil en sus organizaciones.

En este contexto, pues, participativo, digital y en búsqueda de la actitud solidaria de los jóvenes, el discurso de los representantes del Tercer Sector ha puesto de manifiesto que las ONG están ajustando sus estrategias para acercarse de manera más efectiva a los jóvenes. Entre esas estrategias destacan el rejuvenecimiento de sus equipos de comunicación en redes, el recurso a los influenciadores, celebrities, youtubers y bloggers para sus campañas, y en definitiva, su intento constante por adaptarse a un hábitat digital que les ha desbordado pero que reconocen imprescindible para llegar a los jóvenes.

Además, el feedback que consiguen en las redes, tanto ONG como plataformas, les ayuda a enfocar sus campañas de manera mucho más efectiva al poder testar su impacto y corregir, si es necesario, su enfoque.

Las acciones de comunicación de las ONG están orientadas a poner la semilla para crear en el futuro ciudadanos participativos que reflexionen y se impliquen en otras cuestiones de interés social. Con ese fin, recurren en sus campañas a los valores éticos más tradicionales, con los que todo el mundo se puede identificar, tales como la honestidad, la transparencia, la lucha contra la corrupción o la preocupación por los demás. En este sentido, las ONG cumplen con una labor de formación cívicopolítica que debe ofrecer resultados a corto plazo, formando ciudadanos digitales participativos.

\section{Referencias bibliográficas}

Arrieta, E. (2017). Change.org alcanza los 12 millones de usuarios en España. Expansión Recuperado de http://www.expansion.com/economia-digital/companias/ 2017/04/18/58f63935268e3e76658b45d2.html.

Berceruelo, B. (2016). Planificar en Internet. Online u offline: Solo comunicación Empresarial. En B. Berceruelo (coord.), Comunicación empresarial, (pp. 281283). Madrid: Estudios de Comunicación.

Bernal, A. I. (2015). Tecnología, redes sociales, política y periodismo. ¿Pluralidad informativa o efecto bumerán?. Cuadernos.info, 36. doi: 10.7764/cdi.36.647

Brinkerhoff, J., y Brinkerhoff, D. (2002). Government - Nonprofit relations in Comparative Perspective: Evolution, Themes and New Directions. Public Administration and Development, 22(1), 3-18.

Cabanelas, L. (22 de noviembre de 2015). Redes sociales, el «arma de sofá» de las tragedias contemporáneas. ABC. Recuperado de http://www.abc.es/internacional/abci-atentados-paris-redes-sociales-arma-sofa-tragedias-contemporaneas-201511222317_noticia.html 
García, C., y Fernández, C. (2016). Si lo vives, lo compartes. Madrid: Editorial Ariel. García, C., Fernández, C., y Porto, L. (2017). Empoderamiento de los jóvenes a través de las redes sociales. Construcción de una ciudadanía digital comprometida. Comunicación y Sociedad, 31.

González, A. (2015). Tejiendo la red... de influenciadores ¿algo nuevo bajo el sol? Communisensu. Recuperado de https://www.communisensu.com/marketingcomunicacion/tejiendo-la-red-de-influenciadores-algo-nuevo-bajo-el-sol/

González-Anleo, J. (2005). Jóvenes y valores cívico-políticos. Educación y Futuro, $13,59-70$.

Goss, S. (2001). Making local governance work - Networks, Relationships and the Management of Change. New York: Palgrave.

Hernández, R., Fernández, C., y Baptista, P. (2006). Metodología de la Investigación. México: McGraw-Hill.

Kallio K. P., y Häkli, J. (2013). Children and Young People's Politics in Everyday Life. Space And Polity, 17(1). Recuperado de http://www.tandfonline.com/doi/ab s/10.1080/13562576.2013.780710

Kavada, A. (2012). Engagement, bonding, and identity across multiple platforms: Avaaz on Facebook, YouTube, and MySpace. Journal of Media and Communication Research, 52, 28-48

Loader, B. D. (2007). Young citizens in the digital age: Political engagement, young people and new media. Londres: Routledge.

Loader, B. D., Vromen, A., y Xenos, M. A. (2014). The networked young citizen: social media, political participation and civic engagement. Information, Communication \& Society, 17(2). Recuperado de http://www.tandfonline.com/doi/abs/1 0.1080/1369118X.2013.871571

López, G. (ed.) (2005). El ecosistema digital: Modelos de comunicación, nuevos medio y público en Internet. Valencia: Servei de Publicacions de la Universitat de Valencia. Recuperado de http://roderic.uv.es/bitstream/handle/10550/53701/ El_ecosistema_digital_modelos_de_comunic.pdf?sequence $=1$

Lovejoy, K., y Saxton, G. D. (2012). Information, Community, and Action: How Nonprofit Organizations Use Social Media. Journal of Computed-Mediated Communication, 17(3), 337-353.

Mariñas, A. (2016). Las empresas frente al reto 2.0. En B. Berceruelo (coord.), Comunicación empresarial (pp. 283-284). Madrid: Estudios de Comunicación.

Marketingdirecto.com (6 de julio de 2014). El 98\% de las ONGs tienen presencia en las redes sociales. Recuperado de www.marketingdirecto.com/digital-general/ social-media-marketing/el-98-de-las-ongs-tienen-presencia-en-las-redes-sociales

Meriläinen, N., y Vos, M. (2011). Human rights organizations and online agenda setting. Corporate Communications, and International Journal, 16(4), 293 310.

Nonprofit tech for good (2017). “2017 Global NGO”. Online Technology Report. Recuperado de http://www.techreport.ngo/english.html

Observatorio de la Juventud en España (2014). Jóvenes, satisfacción personal, participación asociativa y voluntariado. Recuperado de http://www.injuve.es/sites/ default/files/2016/04/publicaciones/informesondeo_2014-1.pdf

Otte, M. (2010). El crash de la información: Los mecanismos de la desinformación cotidiana. Barcelona: Ariel. 
Sabucedo, J. M., Seoane, G., Ferraces, M. J., Rodríguez, M., y Fernández, C. (1996). La acción política en el contexto supranacional y los marcos de acción colectiva. Revista de Psicología Social Aplicada;.6(3), 103-120.

Santa, L. F. (2015). Un acercamiento al concepto de ecosistema digital. Recuperado de http://www.vallempresa365.com/articulos/marketing/ un-acercamiento-al-concepto-de-ecosistema-digital

Scholte, J. A. (1999). Global Civil Society: Changing the World? University of Warwick, CSGR Working Paper, 31.

Yamamoto, M., Kushin, M., y Dalisay, F. (2015). Social media and mobiles as political mobilization forces for young adults: Examining the moderating role of online political expression in political participation. New Media and Society, 17(6), 880-898. doi: 10.1177/1461444813518390

Zafra，I. (2012). Ecosistema digital. Recuperado de http://www.pmfarma.es/ articulos/1143-ecosistema-digital.html 